\title{
Comparative Study of Radio Propagation and Mobility Models in Vehicular Adhoc Network
}

\author{
Pranav Kumar Singh \\ Assistant Professor \\ Department of Computer \\ Science and Engineering \\ Central Institute of Technology, \\ Kokrajhar, Assam-783370
}

(India)

\author{
Kapang Lego \\ Assistant Professor \\ Department of Computer \\ Science and Engineering \\ NERIST, Nirjuli \\ Arunachal Pradesh (India)
}

\begin{abstract}
VANET (Vehicular Adhoc NETwork) has gained interest all over the world, many projects and research communities are working for the deployment of VANET. The main objective of these projects and researches are to develop protocols and tools to provide adequate wireless communication between vehicles. As we all know that these studies and practices are still simulation based because it's infeasible to use real vehicles for the large scenario in different conditions to develop new algorithms and protocols for VANET. A wireless channel is unsteady and lossy so simulators for VANET require a model of these characteristics. In this paper we have highlighted the need of adequate radio propagation and mobility models for VANET as these two factors play an important role to adopt the protocol suitable for vehicle communication
\end{abstract}

\section{General Terms}

Vehicular Adhoc NETwork, Radio propagation model, Mobility Models

\section{Keywords}

Two Ray Ground, Shadowing, Nakagami, Micro-simulation, Bidirectional coupled Simulation mobility model.

\section{INTRODUCTION}

VANET (Vehicular Adhoc NETwork) is a subset MANET (Mobile Adhoc NETwork). The nodes movements in VANET are predicted as the vehicle moves on road they can't move in random direction unlike in case of MANET i.e. the motion patterns are restricted by road topology.

In VANET, or Intelligent Vehicular Ad-Hoc Networking, defines an intelligent way of using Vehicular Networking. In VANET integrates on multiple ad-hoc networking technologies such as WiFi IEEE $802.11 \mathrm{~b} / \mathrm{g}$, WiMAX IEEE 802.16, Bluetooth, IRA, ZigBee for easy, accurate, effective and simple communication between vehicles on dynamic mobility [20].

Vehicles in VANET act as transceiver and are capable to send, receive and forward the information at the same time while creating a highly dynamic network, which is continuously changing.

Vehicles equipped with wireless communication technologies and acting like computer nodes will be on the road soon and this will revolutionize the concept of travelling. VANETs bring lots of possibilities for new range of applications that will sure increase traveler safety, Enhance traveler mobility, Decrease travelling time, Conserve energy and protect the environment, Magnify transportation system efficiency, Boost on-board luxury but it is not enough many other services can be served by using this technology.
VANET needs high cost and great effort for its deployment. The simulation based study is a feasible and useful alternative for the actual deployment. Simulations studies have to cover all the possibilities that are possible on real road i.e. it has to involve large and heterogeneous scenarios (urban, city, highway, rural).

The mobility models and radio propagation both can significantly affect simulation results. For results to be useful, it is important that the simulated model is as close to reality as possible.

We have to consider all the facts for vehicles in VANET i.e. movement pattern, node density and node velocity as well as fading scenario in cities etc. These factors require an approach that can model it in same fashion as in real.

This paper is an attempt to focus the various possible radio propagation and mobility model available to simulate the VANET and we also highlighted the adequate model suitable in VANET scenario that can help to adopt the protocols or VANET.

The paper covers the applications, challenges related work previously done, radio propagation models with their properties, mobility models with their limitations and advantages in VANET respect and proposed radio propagation and mobility model for VANET.

Figure1 below represents a VANET scenario example.

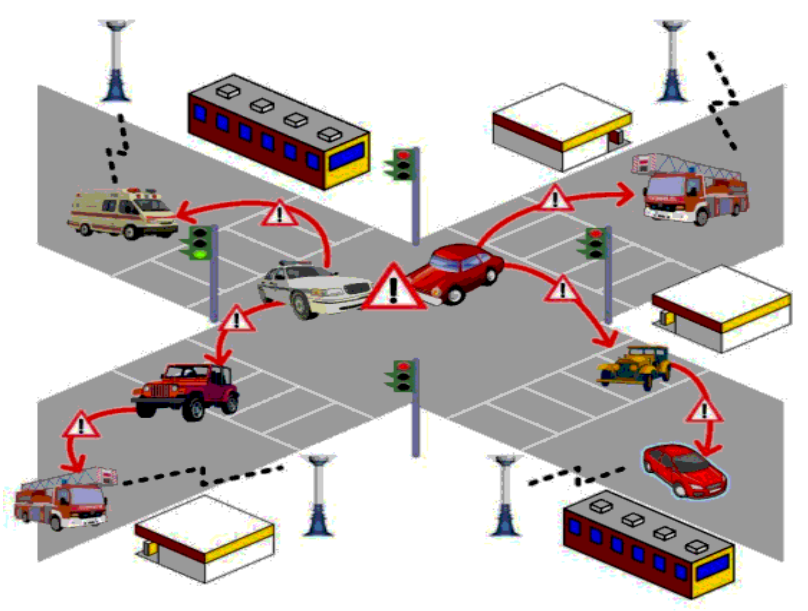

Fig 1: VANET Scenario [2]

\section{VANET APPLICATIONS}

VANETs would support life-critical safety applications, Safety warning applications, electronic toll collection, internet access, automatic parking, roadside service finder, etc.

According to various studies and driver's experiences main applications of VANETs can be divided into two main categories [3, 4, and 5]. 
Safety and

Non-safety

The Table1 given below represents the applications of VANET

Table1. Applications of VANET

\begin{tabular}{|c|c|c|c|}
\hline Type & Description & Example & Communication \\
\hline Safety & $\begin{array}{l}\text { Safety } \\
\text { applications } \\
\text { have the ability } \\
\text { to reduce traffic } \\
\text { accidents and to } \\
\text { improve } \\
\text { general safety. }\end{array}$ & $\begin{array}{l}\text { Safety Critical: The danger is } \\
\text { high or danger is imminent. } \\
\text { Hazardous situations (e.g. like } \\
\text { collisions). } \\
\text { Safety Related: The danger is } \\
\text { either low or elevated, e.g., } \\
\text { curve speed waming, work } \\
\text { zone waming }\end{array}$ & $\mathrm{V} 2 \mathrm{~V}$ or $\mathrm{V} 2 \mathrm{I} / \mathrm{I} 2 \mathrm{~V}$. \\
\hline Non Safety & $\begin{array}{l}\text { These are } \\
\text { applications } \\
\text { that provide } \\
\text { traffic } \\
\text { information and } \\
\text { enhance driving } \\
\text { comfort }\end{array}$ & $\begin{array}{l}\text { Traffic optimization } \\
\text { Traffic information and } \\
\text { recommendations, enhanced } \\
\text { route guidance etc. } \\
\text { Infotainment } \\
\text { The Infotainment services are } \\
\text { Intemet access, media } \\
\text { downloading, instant messaging } \\
\text { etc. } \\
\text { Payment services like } \\
\text { Payment services to collection, } \\
\text { Electronic toll } \\
\text { parking management etc. } \\
\text { Roadside service finder } \\
\text { Finding nearest fuel station, } \\
\text { restaurants etc. This involves } \\
\text { communication of vehicles with }\end{array}$ & $\begin{array}{l}\text { V2I or I2V } \\
\text { communication }\end{array}$ \\
\hline
\end{tabular}

Table2.shows the comparisons between the above application on the bases of priority, latency, and network traffic and message range.

Table2. Comparison of VANETs applications

\begin{tabular}{|l|l|l|l|l|}
\hline Applications & Priority & $\begin{array}{l}\text { Allowable } \\
\text { latency }\end{array}$ & $\begin{array}{l}\text { Network } \\
\text { Traffic }\end{array}$ & $\begin{array}{l}\text { Message } \\
\text { range(m) }\end{array}$ \\
\hline $\begin{array}{l}\text { Safety } \\
\text { Critical }\end{array}$ & Class1 & 100 & Event & $\begin{array}{l}\text { Minimum } \\
250\end{array}$ \\
\hline $\begin{array}{l}\text { Safety } \\
\text { Related }\end{array}$ & Class2 & 100 & Periodic & $\begin{array}{l}\text { Minimum } \\
\text { Non safety }\end{array}$ \\
& Class3 & $50-500$ & Event & $50-250$ \\
& & & & \\
\hline
\end{tabular}

\section{CHALLENGES OF VANET}

Vehicular ad hoc networks behave in different ways than conventional MANETs. Driver behavior, mobility constraints, and high speeds create unique characteristics of VANETs.

These characteristics have important implications for designing decisions in these networks. Thus, numerous challenges need to be addressed for inter-vehicular communications to be widely deployed [5] [6] [7]. Table3 represents some of the main challenges related to VANET.

Table3. Challenges of VANET

\begin{tabular}{|c|c|}
\hline Type & Description \\
\hline $\begin{array}{l}\text { Morement } \\
\text { pattern }\end{array}$ & $\begin{array}{l}\text { City roads: High node density, small roads, Many intersections. } \\
\text { Often, buildings right beside the roads limit wireless } \\
\text { communication. } \\
\text { - Rural roads: Larger segments, intersections are rarer. Few } \\
\text { vehicles, traffic conditions often do not allow the formation of a } \\
\text { connected network. } \\
\text { - Highways: Multi-lane road, very large segments and well-defined } \\
\text { exits and on-ramps. High speed traffic encountered here. A node } \\
\text { can quickly join or leave the network in a very short time leading to } \\
\text { frequent network partitioning and topology changes. These } \\
\text { movement scenarios pose special challenges particularly for the } \\
\text { routing. }\end{array}$ \\
\hline Node Velocity & $\begin{array}{l}\text { High node relocities: The mutual wireless communication } \\
\text { window is very short due to a relatively small transmission range of } \\
\text { several hundred meters. } \\
\text { Slow morement: Stable topology, but a very high vehicle density, } \\
\text { which results in high interference, medium access problems, etc. }\end{array}$ \\
\hline Node Density & $\begin{array}{l}\text { The number of other vehicles in mutual radio range may vary from } \\
\text { zero to dozens or even hundreds. } \\
\text { Low density: Immediate message forwarding gets impossible, } \\
\text { more sophisticated information dissemination is necessary, which } \\
\text { can store and forward selected information, when vehicles } \\
\text { encounter each other. Same message may be repeated by the same } \\
\text { vehicle multiple times. } \\
\text { High density: Here, a message should be repeated only by selected } \\
\text { nodes, because otherwise this may lead to an overloaded channel }\end{array}$ \\
\hline Security [8] & $\begin{array}{l}\text { The content of a received message has to be verified within a short } \\
\text { time to be able to use the information as soon as possible. } \\
\text { Authentication } \\
\text { The authentication service is concerned with assuring that the } \\
\text { communication is authentic in its entities. } \\
\text { Integrity } \\
\text { The integrity service deals with the stability of a stream of } \\
\text { messages. It assures that messages are received as sent, without } \\
\text { modification, insertion, reordering, or replays } \\
\text { Confidentiality } \\
\text { It guarantees the privacy of drivers against unauthonized observers } \\
\text { Accessibility } \\
\text { A kind of attacks can result in the loss or diminution in the } \\
\text { accessibility. Even a robust communication channel can still suffer } \\
\text { some attacks (such as deny of service) which can bring down the } \\
\text { network. Therefore, availability should be also supported by } \\
\text { altemative means. }\end{array}$ \\
\hline Scalability[8] & $\begin{array}{l}\text { The number of users and/or the traffic volume can be increased } \\
\text { with reasonably small performance degradation or even network } \\
\text { outage and without changing the system components and protocols }\end{array}$ \\
\hline
\end{tabular}




\section{RELATED WORK}

We found few and very good studies that have highlighted the use of radio propagation and mobility models in VANET. In this section of our paper we have mentioned some of those research studies.

A research study by Imran Khan and others [9] has analyzed the AODV and OLSR routing protocols performances in highly fading scenario i.e. by using Nakagami propagation model and has focused the limitations of TwoRayGround propagation model. This study doesn't highlight the details of mobility and propagation models used.

Arijit Khan [10] and other members in his graduation project "A comparative analysis of DSRC and 802.11 over Vehicular Ad hoc Networks" has analyzed the performance of DSRC and 802.11 by using Nakagami propagation model in urban and highway for few vehicles, this study fails to give the details of mobility model used.

Christoph Sommer and Falko Dressler [1] in his study have well defined the historical evolution of mobility models in VANET. Though this study hasn't given any importance to radio propagation model but this is good approach and we have also included on our paper details of mobility model defined by them.

Vaishali D. Khairnar and other members [11] in his study has selected mobility model for VANET but this study has only given their idea of the mobility provided by MOVE [12] simulation tools, they didn't cover the mobility model details and also fails to describe the importance of radio propagation model and other mobility model.

\section{RADIO PROPAGATION MODELS}

Radio propagation is the behavior of radio waves when they are transmitted, or propagated from one point on the Earth to another, or into various parts of the atmosphere[13]. Like light waves, radio waves are affected by the phenomena of reflection, refraction, diffraction, absorption, polarization and scattering.

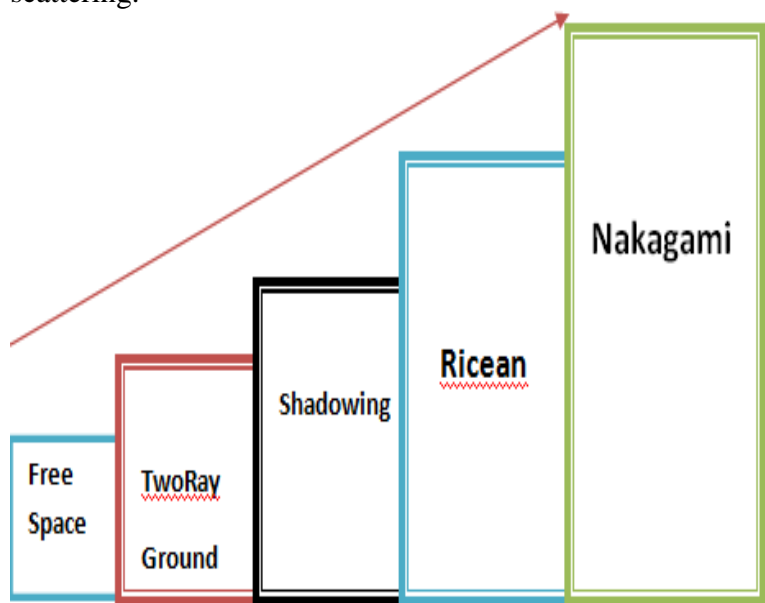

Free space to fading Scenario

Fig 2: Radio Propagation Models Evolution for VANET

Figure2 represents the radio propagation models used and the evolution of it from free space to Nakagami radio propagation for fading scenario.

In this section of paper we tried to cover the details of each radio propagation model with their applicability in VANET.

\subsection{Free Space Radio Propagation}

This radio propagation model is earliest model and was used in MANET by researchers in the earliest studies.

The Free Space model represents by equation (1) a signal propagating through open space, with no environmental effects. It has one parameter, called "lineofsight". With this parameter off, terrain has no effect on propagation. With it on, the model uses terrain data solely to determine if a line-ofsight (LOS) exists between the transmitting and receiving antennas. If there is no LOS, the signal is blocked entirely and no communication takes place [14].

$$
P_{r}=P_{t} G_{t} G_{r} \lambda^{2} /(4 \pi)^{2} d^{2} L-------------(1)
$$

Where $P_{r}$ the received signal is power (in Watt), $P_{t}$ is the transmitted signal power, $G_{r}$ and $G_{t}$ are the gains of the receiving and the transmitting antennas respectively. $\lambda$ is the wave length, $\mathrm{L}$ is the system loss, and $\mathrm{d}$ is the distance between the transmitter and the receiver.

\section{Applicability in VANET}

This propagation model is not suitable for VANET. The received power is only dependent on the transmitted power, the antenna gains and on the distance between the sender and the receiver. Obstacles are not modeled. In VANET vehicles have to move in city where obstacles are main concerns as the buildings alongside the road will block the transmission or communication.

\subsection{TwoRayGround Radio Propagation}

This radio propagation model is highly preferred in MANET by researchers. This radio propagation model is also used in maximum research studies for protocols performances in VANET scenario.

TwoRayGround model is a more realistic than the Free-Space model as it addresses the case when we consider a ground reflected propagation path between transmitter and receiver, in addition to the direct LOS path. This model is especially useful for predicting the received power at large distances from the transmitter and when the transmitter is relatively high above the ground. It is interesting to note that at far distances, the received power becomes independent of the frequency [15].

The received power at distance $\mathrm{d}$ is predicted by:

$$
\mathrm{P}_{\mathrm{r}}=\mathrm{P}_{\mathrm{t}} \mathrm{G}_{\mathrm{t}} \mathrm{G}_{\mathrm{r}} \mathrm{h}_{\mathrm{t}}^{2} \mathrm{~h}_{\mathrm{r}}^{2} / \mathrm{d}^{4} \mathrm{~L}
$$

Where ht and $\mathrm{hr}$ are the heights of the transmitter and receiver antennas respectively. To be consistent with the free space model $\mathrm{L}$ is added here. The above equation shows a faster power loss than Eq. (1) as distance increases i.e. the received power attenuates much more rapidly with distance, compared to the Free-Space model, i.e., attenuates to the fourth power of the distance

\section{Applicability in VANET}

The two-ray model does not give a good result for a short distance due to the oscillation caused by the constructive and destructive combination of the two rays.

This model assumes that the received energy is the sum of the direct line of sight path and the reflected path from the ground. It takes no account for obstacles and sender and receiver have to be on the same height [16]. We can say this model is also not applicable for VANET. 


\subsection{Shadowing Radio Propagation}

This model allows to model many possible scenario that can affect radio signal power for example using path loss value $\beta$ we can set as well as in building scenario of real communication . Research study by Ibrahim khider and others [17] have well defined it:

In this model it is assumed that the average received signal power decreases logarithmically with distance. A Gaussian random variable is added to this path loss to account for environmental influences at the sender and the receiver. The shadowing model consists of two parts. The first one is known as path loss model, which also predicts the mean received power at distance $d$, denoted by $\overrightarrow{\operatorname{Pr}(d)}$. It uses a close-in distance d0 as a reference. $\overrightarrow{\operatorname{Pr}^{(d)}}$ is computed relative to $\mathrm{P}_{\mathrm{r}}(\mathrm{d} 0)$ as follows.

$$
\frac{\operatorname{Pr}(d 0)}{\operatorname{Pr}(d)}=\left(\frac{d}{d 0}\right) \beta
$$

$\beta$ is called the path loss exponent, and is usually empirically determined by field measurement. Table 4 given below gives some typical values of $\beta$ larger values correspond to more obstructions and hence faster decrease in average received power as distance becomes larger.

Table4. Some Typical values of path loss $\beta$

\begin{tabular}{|l|l|c|}
\hline \multicolumn{2}{|c|}{ Environment } & $\boldsymbol{\beta}$ \\
\hline \multirow{2}{*}{ Outdoor } & Free Space & 2 \\
\cline { 2 - 3 } & Shadowed urban area & 2.7 to 5 \\
\hline \multirow{2}{*}{ In building } & Line-of-sight & 1.6 to 1.8 \\
\cline { 2 - 3 } & Obstructed & 4 to 6 \\
\hline
\end{tabular}

Path loss in $\mathrm{dB}$ can be measured by given eq ${ }^{\mathrm{n}} 4$

$$
\left[\overline{\frac{P_{r}(d)}{P_{r}\left(d_{0}\right)}}\right]_{d B}=-10 \beta \log \left(\frac{d}{d_{0}}\right)
$$

The second part of the shadowing model reflects the variation of the received power at certain distance. It is a lognormal random variable that is; it is of Gaussian distribution if measured in $\mathrm{dB}$. The overall shadowing model is represented by

$$
\left[\frac{P_{r}(d)}{P_{r}\left(d_{0}\right)}\right]_{d B}=-10 \beta \log \left(\frac{d}{d_{0}}\right)
$$

Where $\mathrm{XdB}$ is a Gaussian random variable with zero mean and standard deviation $\sigma_{\mathrm{dB}} \cdot \sigma_{\mathrm{dB}}$ is called the shadowing deviation, and is also obtained by measurement. Table 5 shows some typical values of $\sigma_{\mathrm{dB}}$.

Table5. Some Typical values of shadowing deviation $\sigma_{\mathrm{dB}}$

\begin{tabular}{|l|c|}
\hline \multicolumn{1}{|c|}{ Environment } & $\boldsymbol{\sigma}_{\mathbf{d B}}(\mathbf{d B})$ \\
\hline Outdoor & 4 to 12 \\
\hline Office, hard partition & 7 \\
\hline Office, soft partition & 9.6 \\
\hline Factory, line-of-sight & 3 to 6 \\
\hline Factory, obstructed & 6.8 \\
\hline
\end{tabular}

\section{Applicability in VANET}

The shadowing radio propagation model can be used in VANET scenario by varying either $\beta$ or $\sigma_{\mathrm{dB}}$ values to set the various environment conditions for obstacles. This model will help to check the performance of routing protocols in VANET for example setting $\beta$ value to 2.7 to 5 to form shadowed urban scenario. This model is very realistic in case of MANET but in VANET we have only outdoor of highly fading scenario.

\subsection{Ricean and Rayleigh fading models}

These two models are fading models, meaning that they describe the time-correlation of the received signal power. Fading is mostly caused by multipath propagation of the radio waves. If there are multiple indirect paths between the sender and the receiver, Rayleigh fading occurs. If there is one dominant (line of sight) path and multiple indirect signals, Ricean fading occurs [17].

The model behind Ricean fading is similar to that for Rayleigh fading, except that in Ricean fading a strong dominant component is present.

This dominant component can for instance be the line-of-sight wave. Refined Ricean models also consider that that the dominant wave can be a phasor sum of two or more dominant signals, e.g. the line-of-sight, plus a ground reflection. This combined signal is then mostly treated as a deterministic (fully predictable) process, and that the dominant wave can also be subject to shadow attenuation [18]

This signal received over a Ricean multipath channel can be expressed as

$v(t)=C \cos \mathrm{w}_{c} t+\mathrm{S}_{n=1}^{N} \quad \mathrm{r}_{n} \cos \left(\mathrm{w}_{c} t+\mathrm{f}_{n}\right)$

where

$C$ is the amplitude of the line-of-sight component $\mathrm{r}_{n}$ is the amplitude of the $n$-th reflected wave $\mathrm{f}_{n}$ is the phase of the $n$-th reflected wave $n=1 . . N$ identify the reflected, scattered waves.

Rayleigh fading is recovered for $C=0$

\subsubsection{Ricean factor ( $K$-factor)}

The Ricean $K$-factor is defined as the ratio of signal power in dominant component over the (local-mean) scattered power. In the expression for the received signal, the power in the lineof-sight equals $C^{2} / 2$. In indoor channels with an unobstructed line-of-sight between transmit and receive antenna the $K$ factor is between, say, 4 and $12 \mathrm{~dB}$. Rayleigh fading is recovered for $K=0$ (-infinity $\mathrm{dB}$ ).

\section{Applicability in VANET}

Vehicle-to-vehicle data communication will mainly consist of the continuous (routine) exchange of telemetric data such as vehicle status, speed, and acceleration. Interfering signals will be present from vehicles within the platoon and from outside the platoon (from vehicles in other lanes). Vehicles with bumper mounted directional antennas are considered.

The vehicle-to-vehicle radio link can be modeled statistically as a Ricean fading channel by setting large $\mathrm{K}$-factor value as the dominant component in the Ricean fading channel is likely to be relatively strong compared to the reflected signal, 
and the delay spread is likely to be relatively small because reflections occur in the immediate vicinity of the transmitter and receiver antenna [18].

\subsection{Nakagami Radio Propagation}

This radio propagation model is well defined in [19] and given as:

Nakagami is a mathematical general modeling of a radio channel with fading. Compared to the existing models (shadowing and two-ray ground), Nakagami RF model has more configurable parameters to allow a closer representation of the wireless communication channel. It is able to model from an perfect free space channel, to a moderate fading channel on highway, even to a dramatically fading channel in urban communities.

Nakagami distribution is defined by the following probability density function:

$$
f(x)=\frac{2 m^{m} x^{2 m-1}}{\Gamma(m) \Omega^{\mathrm{m}}} \exp \left[\frac{-m x^{2}}{\Omega}\right], x \geq 0, \Omega>0, m \geq \frac{1}{2}
$$

The corresponding pdf (probability density function) of power (square of the signal amplitude) at the given distance can be obtained by a change of variables and is given by a gamma distribution of the following form:

$$
p(x)=\left(\frac{m}{\Omega}\right)^{m} \frac{x^{m-1}}{\Gamma(m)} \exp \left[-\frac{m x}{\Omega}\right], x \geq 0
$$

$\Omega$ is the expected value of the distribution and can be interpreted as the average received power. $m$ is the so called shape or fading parameter.

The values of the parameters $m$ and $\Omega$ are functions of distance. So the Nakagami model is defined by two functions: $\Omega$ (d) and $\mathrm{m}(\mathrm{d})$.

- Rayleigh distribution is a special case of Nakagami distribution where $m(d)=1$ (for every $d$ )

- Larger values of $m$ give less severe fading.

\section{Applicability in VANET}

This model is able to from a perfect free space channel, to a moderate fading channel on highway, even to a dramatically fading channel in urban communities. By varying shape factor $\mathrm{m}$ value we can form highly fading scenario like urban or city as well as free way of highway. This radio propagation model we can say is highly applicable for the use in protocol performances in VANET.

\section{MOBILITY MODELS}

Selection of proper mobility is equally important as selection of suitable radio propagation model for VANET. In this section of our paper we have described the historical evolution of mobility model used inVANET and it is well defined in the study by Christoph Sommer and Falko Dressler [1] and is given as follows:

Simulations of VANET scenarios are concerned with the accurate modeling of single radio wave transmissions between nodes and, therefore, require exact positions of simulated nodes. Only microscopic simulations, which model the behavior of single vehicles and interactions between them, can be considered as an adequate mobility model for simulated VANET nodes [1].
The historical evolution of mobility models used in simulations of VANET protocols and application is illustrated in Figure3.

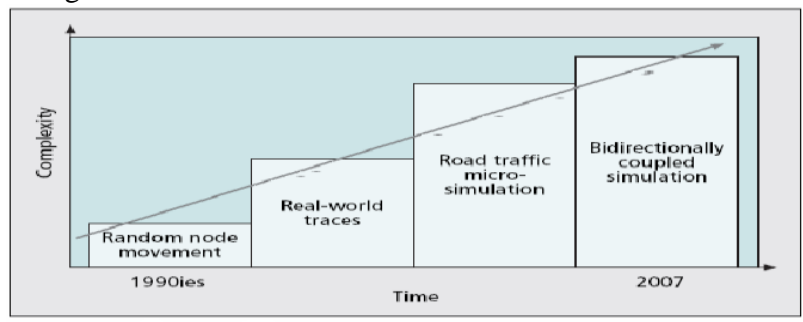

Fig 3: Historical evolution of mobility modeling strategies and techniques in VANET research [1]

\subsection{Random Node Movement}

The earliest model that was highly used in ad hoc network research, for the movement of nodes in an unconstrained, completely random manner, termed the Random Waypoint mobility model (RWM) .This mobility model was highly used by researchers in MANET where node movement is in random direction but this model is not an appropriate choice for VANET as vehicles has defined mobility.

Drawbacks

1. This model was Imprecise

2. Not suitable for VANET

\section{$3 . \quad$ Potentially unstable}

\subsection{Real-World Mobility Traces}

This mobility model was used by tracking the real vehicles using onboard devices and the vehicle positions were recorded at regular interval. These trace files are then stored and subsequently used in network simulations.

Drawbacks

1. Very costly

2. Time consuming process

3. Changing only one parameter, e.g. the density of vehicles, and keeping all other parameters unchanged is simply infeasible in reasonably large scenarios.

\subsection{Road Traffic Micro-Simulation}

This mobility model is based on artificial mobility traces of vehicles on real topology of roads unlike the previous mobility model where real world vehicles movements were used.

This mobility model has the advantage over in terms of free parameterization i.e. various scenarios can be generated by freely changing the parameters.

Draw Back

1. This main drawback of this mobility model is no feedback on driver behavior, i.e. accidental, emergency and hazardous situation on road is not covered.

\subsection{Bidirectionally Coupled Simulation}

This is the most adequate and important simulation methodology and a realistic approach.

The bidirectional coupled simulation form the loop between road traffic and network traffic simulation is very useful in situations where accident information, danger warning and traffic congestion information is relayed, which results in altering driver's behavior.

Drawback

1. This model is not yet stable; research communities are still working on it. 


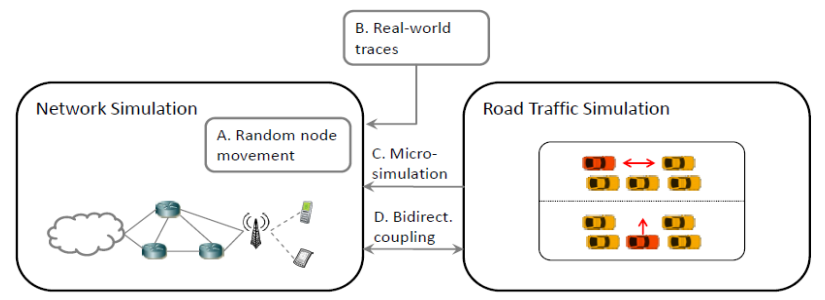

Fig 4: Mobility modeling techniques for simulation of VANET protocols and applications [1].

Figure4 represents the scenario of mobility models used in VANET defined in [1]

\section{PROPOSED RADIO PROPOGATION AND MOBILITY MODEL FOR VANET}

We after this study found that the Nakagami is suitable and realistic radio propagation model for VANET and the bidirectional coupled simulation approach of mobility model will help to form realistic scenario of VANET and can be used for various applications like hazardous and accident scenario on road. By using these two factors in proper manner the protocol performances will be accurate and help the organizations to adopt the better protocol for VANET.

\section{CONCLUSION}

This paper has highlighted various radio propagation models and mobility models and explained their features in respect to VANET. Most of these radio propagation models are now bundled with network simulator ns-2 and some simulation tools are also available for VANET like MOVE, TraNS and Veins that can help researchers to check the performance of routing protocols using these proposed and appropriate models for VANET.

\section{REFERENCES}

[1] Sommer, C.; Dressler, F.; Univ. of ErlangenNuremberg, Erlangen, "Progressing Towards Realistic Mobility Models in VANET Simulations" appears in: Communications Magazine, IEEE Issue Date: November 2008 ,Volume: 46 Issue:11 On page(s): 132 - 137

[2] Mobile Networking Lab: available at http://monet.postech.ac.kr/

[3] Vehicle Safety Communications Project Task 3 Final Report. Technical report, The CAMP Vehicle Safety Communications Consortium, Mar 2005. Sponsored by U.S. Department of Transportation (USDOT). Available through National Technical Information Service, Springfield, Virginia 22161.

[4] Rainer Kroh, Antonio Kung, and Frank Kargl. VANETS Security Requirements Final Version. Technical report, Secure Vehicle Communication (Sevecom), Sep 2006. Availablel http://www.sevecom.org/Pages/ProjectDocuments.html.

[5] Elmar Schoch, Frank Kargl, Michael Weber, and Tim Leinmuller. Communication Patterns in VANETs. IEEE Communications Magazine, 46:119-125, Nov 2008.

[6] Moez Jerbi, Rabah Meraihi, Sidi-Mohammed Senouci, and Yacine Ghamri-Doudane.An Improved Vehicular Ad Hoc Routing Protocol for City Environments. IEEE International Conference on Communications (ICC ’07),pages 3972-3979, 2007.
[7] A. Stampoulis and Z. Chai. Survey of Security in Vehicular Networks. Technical report, 2007. Project CPSC 534.

[8] P a g e | 72 Vol. 10 Issue 7 Ver. 1.0 September 2010 Global Journal of Computer Science and Technology "VANET Parameters and Applications: A Review "Kamini1, Rakesh, Kumar2.

[9] Khan, I. Qayyum, A. Center of Res. in Networks \& Telecom (CoReNeT), M.A. Jinnah Univ., Islamabad, Pakistan, "Performance evaluation of AODV and OLSR in highly fading vehicular ad hoc network environments" appears in Multitopic Conference, 2009. INMIC 2009. IEEE $13^{\text {th }}$,International Issue Date: 14-15 Dec. 2009 On page(s): $1-5$

[10] Arijit Khan, Shatrugna Sadhu, and Muralikrishna Yeleswarapu ,Dept. of Computer Science, University of California, Santa Barbara "A comparative analysis of DSRC and 802.11 over Vehicular Ad hoc Networks" submitted project in graduation.

[11] Vaishali D. Khairnar Symbiosis Institute of Technology Pune, Dr. S.N.Pradhan

Institute of Technology, Nirma University, Ahmedabad, "Mobility Models for Vehicular Ad-hoc Network Simulation" International Journal of Computer Applications (0975 - 8887) Volume 11- No.4, December 2010.

[12] F. Karnadi, Z. Mo, K.-C. Lan, "Rapid Generation of Realistic Mobility Models for VANET", in Proc. of the IEEE Wireless Communication and Networking Conference (WCNC" 07), March 2007.

[13]H. P. Westman et al., (ed), Reference Data for Radio Engineers, Fifth Edition, 1968, Howard W. Sams and Co., no ISBN, Library of Congress Card No. 43-14665 page 26-1

[14] Mohammad Siraj \& Soumen Kanrar "Performance of Modeling wireless networks in Realistic environment" International Journal of ComputerNetworks (IJCN), Volume (2): Issue (1) 62-79

[15] Masood Khosroshahy, Thierry Turletti, Katia Obraczka, "Snapshot of MAC, PHY and Propagation Models for IEEE 802.11 in Open-Source Network Simulators",Project Report, September2007,INRIA

[16] Martinez, F.J.; Chai-Keong Toh; Cano, J.-C.; Calafate, C.T.; Manzoni, P.; Univ. of Zaragoza, Zaragoza "Realistic Radio Propagation Models (RPMs) for VANET Simulations" appears in: Wireless Communications and Networking Conference, 2009. WCNC 2009. IEEE Issue Date: 5-8 April 2009 On page(s): 1 - 6

[17] Ibrahim khider_, Furong Wang, WeiHua Yin, Sacko, "The impact of different radio propagation models for Mobile Ad-hoc NETworks (MANET) in urban area environment" World Journal of Modelling and Simulation Vol. 5 (2009) No.1, pp. 45-52, England,UK

[18] Vehicle to Vehicle RF Propagation Measurement http://www.wirelesscommunication.nl/reference/chaptr0 3/ricepdf/rice.htm

[19] Documentation - CODE README, "Overhaul of IEEE 802.11 Modeling and Simulation in NS-2 (802.11Ext) " Available at: dsn.tm.uka.de/medien/.../DocumentationNS-2-80211Ext-2008-02-22.pdf

[20] The Theory of Vehicular Ad-Hoc Network". TechViewz.Org., 2008-02 http://techviewz.org/2008/02 /theory-of-vehicular-ad-hoc-network.html. Retrieved 2010-10-18 